dem Object $\boldsymbol{B}$, das sich allerdings noch mehr als $A$ verandert hat. Mit seiner Bewegung käme man auf Anfang October 1900, was wohl unmöglich ist. Es bleiben die Möglichkeiten, dass entweder die Geschwindigkeiten in diesem Theil der Hülle stark abgenommen haben, oder dass die zweite Welle (Hülle) hier gemessen ist. Die Form der Hülle würde beidem entsprechen. Dieselbe liegt, wie ja auch die Zahlen zeigen, bei $A$ weiter von der Nova entfernt.

Man kann nun nicht viel falsch gehen, wenn man annimmt, dass die Erscheinung den Weg von der Nova bis zum Punkt $A$ mit gleichförmiger Geschwindigkeit durcheilt hat. Sagen wir, dass das Object $A$ den Weg von $380^{\prime \prime}$ in rund 180 Tagen zurückgelegt hat, damit es vom Moment der Entstehung der Nova bis zum 23. August diesen Weg durcheilte. Es würde dies in einer Entfernung, die der Parallaxe von 61 Cygni (Struve) entspräche, mehr als $7000 \mathrm{~km}$ Geschwindigkeit pro Secunde verlangen.

Der Lichtgeschwindigkeit - bei der Fortbewegung von

Heidelberg, I 901 Nov. 29.
$A$ - entspräche eine Parallaxe von etwa ०."० ı, während der Stern mindestens zehnmal näher sein müsste als 6 i Cygni, wenn man plausible Protuberanzengeschwindigkeiten erhalten wollte. Eine solche Parallaxe müsste sich sehr bald zeigen. Es scheinen also vorerst wohl die Geschwindigkeit der elektrischen Welle und die Parallaxe von o."ora am wahr. scheinlichsten zu sein.

Leider verhindert das schlechte Wetter weitere Aufnahmen, deshalb theile ich die obigen Messungen vorläufig mit.

Die optische Hülle, die Flammarion und Antoniadi entdeckt haben, hat sich, wie bereits gemeldet, ebenfalls verändert. Diese Aureole ergab die folgenden Durchmesser:

$\begin{array}{ccc}\text { Igor } & \text { Bruce Teleskop } a & \text { Bruce Teleskop } b \\ \text { Aug. } 23 & 35^{\prime \prime} & 3^{8} 3^{\prime \prime} \\ \text { Nov. } 17 & 3 \text { 19 } & 35^{2}\end{array}$

Die Lichtart hat sich also stark verändert.

Max Wolf.

\title{
Notiz betr. die Eigenbewegung der Nova Persei (Ch. 1226).
}

Am 12. October habe ich, correspondirend zu den zwei Beobachtungen im Frühjahr (A. N. 3726), eine Ortsbestimmung der Nova Persei am Repsold'schen Meridiankreis ausgefuhrt, fand aber keine nennenswerthe E. B., so dass ich weitere Messungen zunächst unterliess. Auf die Bemerkung

\begin{tabular}{|c|c|}
\hline $\begin{array}{l}\text { 1901 } \\
\text { Febr. } 26 \\
\text { März } 13\end{array}$ & $\begin{array}{r}\alpha 1901.0 \\
3^{\mathrm{h}} 24^{\mathrm{m}} 28.15 \\
28.11\end{array}$ \\
\hline Oct. I 2 & 28.17 \\
\hline Nov. 17 & 28.15 \\
\hline
\end{tabular}

Als Vergleichsterne dienten stets $\delta$ Persei und $\nu$ Persei. Da die Theilfehler des Kreises sehr gering sind, so ist die Aenderung der Klemmlage kaum von Bedeutung. In RA.

Königstuhl, 190 I Nov. 20. des Herrn Bergstrand in A. N. 3748 hin beobachtete ich den Stern wieder am 17. November, erhielt aber nur eine Bestätigung des ersten Resultats.

Zur besseren Uebersicht theile ich hier auch die zwei früheren Werthe nochmals mit:

$$
\left.\begin{array}{r}
8 \text { 1901.0 } \\
+43^{\circ} 33^{\prime} 53^{\prime \prime 6} \\
53.8 \\
53.7 \\
53.7
\end{array}\right\} \text { Kl. W. }
$$

wäre hiernach eher eine positive, in Decl. gar keine E. B. vorhanden. Jedenfalls werde ich bei Gelegenheit die Beobachtungen wiederholen.

\section{Notiz betr. 9 Orionis. *)}

Bezugnehmend auf die Mittheilung über $\vartheta$ Orionis in Nr. $375 \mathrm{I}$ kann ich bestätigen, dass am 3 . November am 7 Zöller bei sehr guter Luft und Vgr. I 76 auch hier die Sterne $E$ und $F$ mit einer Klarheit gesehen wurden, wie früher niemals, so dass $F$ selbst einem ungeübten Auge nicht hätte entgehen können. Ich glaubte damals den Effect der reinen und ruhigen Luft zuschreiben zu sollen.

$$
\text { Landstuhl, } 190 \mathrm{r} \text { Nov. } 30 .
$$

\section{Ph. Fauth.}

*) Dr. Hartwiig konnte am 12. November bei einer auf meine Bitte angestellten Prüfung des Trapezes im Orionnebel nichts Auffallendes constatiren. Allerdings war die Luft sehr schlecht und die Anwendung stärkerer Vergrösserungen daher ausgeschlossen. $K r$.

Aufforderung betr. Beobachtungen des Cometen 1900 II. Fohn M. Poor, Princeton University, Princeton, N. Y. (U.S. A.) desire to compute a definitive orbit of Comet I900 II (Borrelly-Brooks) discovered July 23, 1900, and would be glad to receive any unpublished observations.

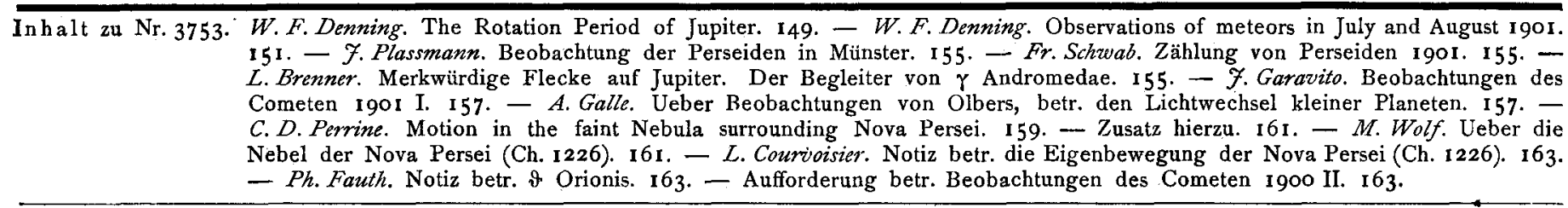

Geschlossen rgor Dec. I2. Herausgeber: H. Kreutz. Druck von C. Schaidt. Expedition: Kiel, Niemannsweg ro3 